\title{
Childhood cancer in Côte d'Ivoire, 1995 - 2004 - challenges and hopes
}

J J A Yao, L Couitchere, Y Atimere, D Koné, R Azagoh-Kouadio, M S Oulai, D C Stefan

Department of Pediatrics, University Hospital of Treichville, Abidjan, Côte d'Ivoire

J J A Yao, MD

L Couitchere, MD

R Azagoh-Kouadio, MD

M S Oulai, MD

Department of Haematology, University Hospital of Treichville, Abidjan, Côte d'Ivoire

Y Atimere, MD

D Koné, MD

Department of Paediatrics and Child Health, Tygerberg Hospital and Stellenbosch University, Parow, Cape Town, Western Cape D C Stefan, MD, PhD

Corresponding author: D C Stefan (cs@sun.ac.za)

\section{Background. There is insufficient research into the state of paediatric oncology in African countries.}

Objectives. The purpose of this study was to analyse the state of paediatric oncology between 1995 and 2004 in Côte d'Ivoire.

Methods. This retrospective descriptive study analysed all patients under the age of 18 who were diagnosed with cancer in Côte d'Ivoire over a period of 10 years (January 1995 - December 2004) with regard to demographics, types of pathology, delay in diagnosis and treatment, treatment modalities, abandonment of treatment and survival rate.

Results. Of 405 patients diagnosed with cancer, 331 were included in the study. Burkitt's lymphoma was the most common malignancy (73.6\%), followed by nephroblastoma (14.5\%) and acute leukaemia (4\%). Delay in diagnosis occurred in $38.7 \%$ of cases and ranged from 1 to 3 months; the average delay from diagnosis to starting treatment was 18 days. An abdominal mass and swelling of the jaw were the most common clinical presentations. Almost half of the patients (48.6\%) were lost to follow-up and over a third (39.3\%) died shortly after admission owing to advanced disease. The overall survival rate was $9.4 \%$.

Conclusions. Cancer in children in Côte d'Ivoire was dominated by Burkitt's lymphoma. The rate of loss to follow-up of almost $50 \%$ is grounds for concern. The overall survival rate of $9.4 \%$ is very low, but such figures are not uncommon for African countries. Collaboration within the Franco-African Group of Paediatric Oncology has contributed to improving the management of children with cancer.

S Afr Med J 2013;103(2):113-115. DOI:10.7196/SAMJ.6365

Childhood cancer is relatively rare, representing only about $1 \%$ of all malignant disease. However, the number of children with cancer in the world is expected to increase in parallel with the global population. Of the estimated 250000 children diagnosed with cancer every year, about 200000 live in countries with limited resources; their survival rate is estimated to be $25 \%$, while in developed countries it approximates $80 \%{ }^{1}$

It is estimated that there are 50000 new cases of childhood cancer every year in Africa. ${ }^{2}$ In countries bordering the Mediterranean sea and in South Africa (SA) leukaemias are the most frequent cancers in children, while in sub-Saharan Africa lymphomas are predominant, with Burkitt's lymphoma (BL) the most common..$^{3-5}$ Data on survival rates in Africa are scarce and the available information is not reassuring. Published 5-year survival rates for BL vary from $5 \%$ to $70 \%,{ }^{6}$ with most countries falling within the lower half of the range.

All efforts to improve outcomes in childhood cancer should be based on a comprehensive and reliable statistical analysis of the situation. In the absence of national cancer registries, let alone national paediatric cancer registries (South Africa has the only such registry on the continent ${ }^{5}$ ), hospital registries may provide the required information.

The aim of this study was to analyse the data entered in the register of the paediatric haematology oncology unit in Treichville, Côte
d'Ivoire, from January 1995 to December 2004, as a starting point for establishing a strategy to improve the situation of paediatric oncology and to evaluate progress over time. The information was grouped under patient demographics, types of cancer, delay in diagnosis and treatment, modalities of treatment, rate of abandonment of treatment, and survival rate.

Côte d'Ivoire is a Francophone African country situated on the western part of the continent, with a population of more than 20 million. Children (under 18 years of age) represent $45 \%$ of the population. About $43 \%$ of all inhabitants live below the poverty line, with the annual gross domestic product per capita being US $\$ 1600$. There are 14 doctors per 100000 people. ${ }^{8}$ Abidjan, with a population of 3310500 , is Côte d'Ivoire's largest city and commercial and banking centre.

The haematology oncology unit of the Department of Paediatrics was set up in Treichville (a commune of Abidjan) in 1995. It has 20 beds and serves as the referral service for all children diagnosed with cancer in Côte d'Ivoire. It also receives patients from neighbouring countries (Mauritania, Senegal, Mali, Burkina Faso, Guinea and Liberia). The service has a radiology facility including a computed tomography (CT) scanner and a paediatric surgery division. Not all cases of childhood cancer over the study period were histologically confirmed, as there were only 
10 pathologists in the entire country and only 3 in the University Hospital of Treichville in Abidjan.

Chemotherapy was not freely available, the cost of management having to be borne by the patients' families, who often could not afford to pay for either the investigations or the medication.

The time period analysed ends just before Treichville joined the GFAOP (Groupe Franco-Africain d'Oncologie Pédiatrique - FrancoAfrican Group of Paediatric Oncology). Treichville became a therapy research unit after joining GFAOP, and results obtained after 2004 will constitute a comparative analysis.

\section{Patients and methods}

A retrospective descriptive study of the Treichville paediatric haematology oncology unit's experience was undertaken. All records of children (0 - 18 years of age) admitted with a malignancy from 1 January 1995 to 31 December 2004 were reviewed. Of 405 patients who presented to the unit during this time period, 331 were included in the study (the remaining folders were incomplete or the diagnosis was not confirmed).

Variables analysed included age at diagnosis, gender, urban or rural area of residence, clinical presentation, stage of disease, delay in diagnosis (defined as the time interval from the onset of symptoms to diagnosis), delay in initiation of treatment following diagnosis, loss to follow-up, abandonment of therapy, therapeutic protocols and survival.

Staging of BL consisted of chest radiography, abdominal ultrasound, bone marrow aspiration (BMA) and trephine biopsy, together with laboratory investigations, including cerebrospinal fluid examination. Patients had CT scans and all cancers were confirmed by biopsy and histopathology.

Staging of nephroblastoma was based on abdominal ultrasound, intravenous pyelography and chest radiography.

In acute leukaemia, investigations undertaken included a full blood count with smear, BMA and trephine biopsy. Flow cytometry and cytogenetic studies were not available, nor were more sophisticated investigations such as magnetic resonance imaging, positron emission tomography or meta-iodobenzylguanidine scans.

Cure was defined as a remission lasting over 2 years ( 1 year for nephroblastoma).

Ethical approval for the study was obtained from the local ethics committee of the University Hospital of Treichville.

\section{Results}

The results of 331 children admitted with a malignancy during the study period were analysed. Of these, 244 (73.6\%) were diagnosed with BL and presented with an abdominal mass, 48 patients with nephroblastoma (5.7\%) constituted the second most common group, 13 patients (4.5\%) were diagnosed with acute leukaemia, and 7 (3.9\%) had Hodgkin's disease. The remaining patients had retinoblastoma (6 cases), rhabdomyosarcoma (5 cases), neuroblastoma (3 cases), and sacrococcygian teratoma, leiomyosarcoma, germ cell tumour, haemangioma of the liver and undifferentiated abdominal sarcoma (1 case each).

The prevalence of childhood malignancies among the paediatric admissions at Treichville doubled over the study period, from $0.32 \%$ in 1995 to $0.76 \%$ in 2004 . Table 1 describes the four most common malignancies in the study, which represent $96 \%$ of all cases.

The sex ratio was 1.35:1 (males 57\%, females 43\%). Most of the patients $(78 \%)$ lived in urban areas and were between 5 and 10 years of age (mean 7.47 years). The most common clinical presentation was an abdominal mass (152 cases, $45.9 \%$ ), followed by swelling of the jaw (109, 32.9\%); 22 patients (6.6\%) had both an abdominal mass and swelling of the jaw at presentation.

Only $76 \%$ of the patients received treatment. More than half (52.6\%) of those who were not treated simply could not afford the costs, while the remainder died before treatment could be started. The chemotherapy protocol used for BL was COPAD modified,

Table 1. Demographics, staging and outcome of 331 children with the most frequent cancers

\begin{tabular}{|c|c|c|c|c|}
\hline Variable & Burkitt's lymphoma & Nephroblastoma & Leukaemia & Hodgkin's lymphoma \\
\hline Number of patients, $n(\%)$ & $244(73.6)$ & $48(5.7)$ & $13(4.5)$ & $7(3.9)$ \\
\hline Females, \% & 38.9 & 56.2 & 46.1 & 28.6 \\
\hline Males, \% & 61.1 & 43.7 & 53.8 & 71.4 \\
\hline Median age, years & 7.4 & 4.1 & 10.2 & 8.4 \\
\hline \multicolumn{5}{|l|}{ Staging, $n(\%)$} \\
\hline I & $14(5.7)$ & $0(0)$ & & \\
\hline II & $12(4.9)$ & $8(16.7)$ & & \\
\hline III & $119(48.8)$ & $9(18.7)$ & & \\
\hline IV & $25(10.2)$ & $2(4)$ & & \\
\hline $\mathrm{V}$ & - & $0(0)$ & & \\
\hline Not staged & $74(30.3)$ & $29(60.4)$ & & 7 \\
\hline \multicolumn{5}{|l|}{ Outcome, \% } \\
\hline Cured & 6.6 & 27.1 & 7.7 & 0 \\
\hline Still treated & 0.8 & 0 & 0 & 0 \\
\hline Lost to follow-up & 50 & 39.6 & 46.1 & 85.7 \\
\hline Relapsed & 2 & 0 & 7.7 & 14.3 \\
\hline Died & 40.6 & 33.3 & 38.5 & 0 \\
\hline
\end{tabular}


consisting of cyclophosphamide $300 \mathrm{mg} / \mathrm{m}^{2}$ on days $1,2,4$ and 5 , vincristine $1.5 \mathrm{mg} / \mathrm{m}^{2}$ on days 3 and 6 , adriamycin $50 \mathrm{mg} / \mathrm{m}^{2}$ and prednisone $60 \mathrm{mg} / \mathrm{m}^{2}$ on days $1-7$, and methotrexate given intrathecally in a dose of $15 \mathrm{mg}$ on days 1 and 7. Induction comprised 3 courses every 2 weeks or as soon as the blood count allowed administration of chemotherapy, with consolidation of 4 monthly courses. The treatment lasted 8 months and the patient was considered cured after achieving complete remission without a relapse for more than 2 years. Patients were followed up every 2 - 3 months during the first year after treatment was completed, every 6 months in the second year and then once a year. Almost half of the patients with BL died (40.6\%), 50\% were lost to follow-up, $2 \%$ relapsed and only $6.6 \%$ were considered cured $(0.8 \%$ were still on treatment at the time of the study).

Of the patients with nephroblastoma, $22.9 \%$ had stage III or IV disease, and more than $60.5 \%$ were not staged. They were treated according to the SIOP 2001 protocol.

Almost a third of the patients with nephroblastoma died, 39.6\% were lost to follow-up, and $27.1 \%$ were still alive a year after treatment.

In $61 \%$ of cases there was a delay in diagnosis of over 1 month. Therapy was instituted within 14 days in $59 \%$ of the children treated. Delays in commencing treatment were due to waiting for laboratory results or histological confirmation, and the families needing time to collect the money needed to pay for the chemotherapy.

Almost half of the patients were lost to follow-up after the first admission and $39.2 \%$ died shortly after the onset of treatment. Only $9.4 \%$ (31 patients) survived in remission for longer than 2 years, a small number suffered a relapse $(2.1 \%)$, and $0.6 \%$ were still on treatment.

\section{Discussion}

The number of children with cancer increased in Côte d'Ivoire over the study period, mirroring experience in the rest of Africa and the world. Malignancy in children in West Africa remains dominated by BL and nephroblastoma. The survival rate for these two malignancies, which are easily diagnosed and respond to chemotherapy, exceeds $90 \%$ in developed countries. The 1-year survival rate for nephroblastoma in this study was $27.1 \%$. The cost of treatment for both cancers is also relatively low compared with that of treating other malignancies: BL treatment costs around US $\$ 50^{9}$ and the cost of treating stage 2 - 3 nephroblastoma, without radiotherapy, amounts to US\$699.10

Other countries from sub-Saharan Africa report a distribution of malignant pathology in children similar to that described in this study. Lymphomas represented $41.2 \%$ of all malignancies in children at the University Teaching Hospital in Enugu, Nigeria, between 1999 and 2004; $;^{11}$ similarly, in the Tanzanian cancer registry between 1973 and 1995, lymphomas were the most frequent childhood malignancy at $38.9 \% .^{12}$ A different situation is reported from countries in northern Africa ${ }^{3,13}$ and SA, ${ }^{5}$ where childhood leukaemias are most frequent.

In contrast to the major improvement in survival rates for childhood cancer in high-income countries and even in Africa, ${ }^{14}$ the overall survival rate for Ivoirian children remained low over the period studied. However, marked increases in survival for children with cancer in low-resource settings have been achieved elsewhere. For instance, in most countries in Central America that have established twinning programmes and collaborative networks, the survival rate of children with acute lymphoblastic leukaemia has risen from less than $20 \%$ to over $60 \%$ over the past decade. ${ }^{15}$

The urban residence of over $75 \%$ of our patients, in contrast to an urbanisation level of around $40 \%$ for the Ivoirian population as a whole, suggests that a number of children with cancer from rural areas did not reach the hospital in Treichville. Another major hurdle on the path to treatment was the need to pay for chemotherapy. This explains why a considerable number of patients were not treated, or abandoned their treatment. The need to raise money for treatment also contributed to the delay between diagnosis and the beginning of therapy. Similar effects of such economic constraints have been described in detail in a study from Nigeria. ${ }^{16}$

In 2004 the Paediatric Hematology Oncology Unit in Cote d'Ivoire joined the GFAO group. ${ }^{17}$ As a result, chemotherapy is now free of charge, treatment follows established protocols, and access to radiological investigations has been opened. This progress in management of paediatric malignancies will be the subject of a future study.

The present study makes available for the first time a synthesis of the data from the archives of the paediatric haematology oncology unit at the University Hospital of Treichville, Côte d'Ivoire. It contributes to completing the picture of the numerous challenges, many of them socio-economic, encountered in the management of children with cancer in Africa. Its main weakness, in common with all retrospective studies, is the often incomplete recording of data together with the loss of records of some patients.

\section{Conclusions}

During its first 10 years of existence, the paediatric haematology oncology unit at Treichville admitted more than 400 children with various malignancies from Côte d'Ivoire and surrounding countries. The most frequent diagnosis in these children was BL, followed by nephroblastoma. There was a high rate of abandonment of therapy, mainly for financial reasons, resulting in a low rate of cure of only $6.6 \%$ for BL and $27.1 \%$ for nephroblastoma (the latter at 1 year of follow-up). The unit's collaboration with the GFAO in 2004 has resulted in a progressive improvement in patient management, and these more recent outcomes will be reviewed in a future study.

\section{References}

1. Kellie SJ, Howard SC. Global child health priorities: What role for paediatric oncologists? Eur I Cance 2008;44:2388-2396. [http://dx.doi.org/10.1016/.ejca.2008.07.022]

2. Stefan DC, Harford J, Stones D, Newton R, Rodriguez-Galindo C. Hope for African children with cancer: African pediatric oncology group. Pediatr Blood Cancer 2012;59(2):349. [http://dxdol org/10.1002/pbc.24069]

3. Missaoui N, Khouzemi M, Landolsi H, et al. Childhood cancer frequency in the center of Tunisia Asian Pac J Cancer Prev 2011;12(2):537-542.

4. Ocheni S, Bioha FI, Ibegbulam OG, Emodi II, Ikefuna AN. Changing pattern of childhoo malignancies in Eastern Nigeria. West Afr J Med 2008;27(1):3-6.

5. Stefan DC, Stones DK. The South African pediatric tumour registry - 25 years of activity. S Afr Med J 2012;102(7):605-606.

6. Hadley LGP, Rouma BS, Saad-Eldin Y. Challenge of pediatric oncology in Africa. Semin Pediatr Surg 2012;21:136-141. [http://dx.doi.org/10.1053/.jsempedsurg.2012.01.006]

7. Central Intelligence Agency. The World Factbook https//wwwcia gov/library/publications/theworld-factbook/ (accessed 23 July 2012).

8. World Health Organization. World Health Statistics 2012. www.who.int/gho/publications/world health_statistics/2012/en (accessed 23 July 2012).

Thseling PB, Molyneux E, Tchintseme F, et al. Treating Burkitt's lymphoma in Malawi, Cameroon an . [http://dx.doi.org/10.1016/S1470-2045(08)70139-6]

10. Stefan DC. How should we treat nephroblastoma in sub-Saharan Africa? Paper presented at the SIOP Nephroblastoma Working Group, 9-10 June 2012 Rome.

11. Ocheni S, Okafor CO, Emodi IJ, et al. Spectrum of childhood malignancies in Enugu, Nigeria (1999 2004). Afr J Med Med Sci 2005;34(4):371-375.

12. Carneiro PM, Kalaokola FM, Kaaya EE. Pediatric malignancies in Tanzania. East Afr Med J 1998;75(9):533-535.

13. Hosny G, Elkaffas SM. Patterns in the incidence of pediatric cancer in Alexandria, Egypt, from 1972 to 2001. J Egypt Public Health Assoc 2002;77(5-6):451-468

14. Stefan DC, Shalongo S, Ribeiro R. Twinning in paediatric oncology - an African experience. S Aff Med J $2011,102(1) 28-29$

15. Howard SC, Marinoni M, Castillo L, et al. Improving outcomes for children with cancer in low-income countries in Latin America: a report on the recent meetings of the Monza International School of Pediatric Hematology/Oncology ( http://dx.doi.org/10.1002/pbc.21003

16. Meremikwu MM, Ehiri JE, Nkanga DG, Udoh EE, Ikpatt OF, Alaje EO. Socioeconomic constraint to effective management of Burkitt's lymphoma in south-eastern Nigeria. Trop Med Int Healt 2005;10(1):92-98. [http://dx.doi.org/10.1111/j.1365-3156.2004.01348.x]

17. Harif M, Barsaoui S, Benchekroun S, et al. Traitement des cancers de lenfant en Afrique: résultat préliminaires du groupe franco africain doncologie pédiatrique. Arch Pediatr 2005;12:851-853.

Accepted 10 August 2012. 